\title{
Intravascular Delivery of Particulate Systems: Does Geometry Really Matter?
}

\author{
Paolo Decuzzi, ${ }^{1,4,6}$ Renata Pasqualini, ${ }^{3}$ Wadih Arap, ${ }^{3}$ and Mauro Ferrari ${ }^{2,5}$
}

Received March 7, 2008; accepted July 24, 2008; published online August 20, 2008

\begin{abstract}
In cancer therapy and imaging, the systemic passive delivery of particulate systems has relied on the enhanced permeability and retention (EPR) effect: sufficiently small particles can cross the endothelial fenestrations and accumulate in the tumor parenchyma. The vast majority of man-made particulates exhibit a spherical shape as a result of surface energy minimization during their synthesis. The advent of phage display libraries, which are revealing the extraordinary molecular diversity of endothelial cells, and the development of processes for fabricating particles with shapes other than spherical are opening the path to new design solutions for systemically administered targeted particulates. In this paper, the role of particle geometry (i.e., size and shape) is discussed at the tissue and cellular scales. Emphasis is placed on how the synergistic effect of particle geometry and molecular targeting can enhance the specificity of delivery. The intravascular delivery process has been broken into three events: margination, firm adhesion and control of internalization. Predictions from mathematical models and observations from in-vitro experiments were used to show the relevance of particle geometry in systemic delivery. Rational design of particulate systems should consider, beside the physico-chemical properties of the surface coatings, geometrical features as size and shape. The integration of mathematical modeling with in-vitro and in-vivo testing provides the tools for establishing a rational design of nanoparticles.
\end{abstract}

KEY WORDS: geometry; nanoparticles; rational design; systemic delivery.

\section{INTRODUCTION}

Organic, inorganic and biological particulate systems are being developed by several laboratories for different biomedical applications ranging from early disease detection to imaging, therapy and follow-up (1-3). Particulate systems are sufficiently small to be administered at the systemic level and transported by the blood flow along the circulatory system can reach potentially any tissue and organ (biological target) within the host. Differently from freely administered drug molecules and monoclonal antibodies, particles can be engineered to carry a large number of drug molecules and contrast agents providing simultaneously a therapeutic and imaging function (particle

\footnotetext{
${ }^{1}$ School of Health Information Sciences, The University of Texas Health Science Center, Houston, Texas 77030, USA.

${ }^{2}$ The Brown Foundation Institute of Molecular Medicine, The University of Texas Health Science Center, Houston, Texas 77030, USA.

${ }^{3}$ Department of Genitourinary Medical Oncology and Department of Cancer Biology, The University of Texas M. D. Anderson Cancer Center, Houston, Texas 77030, USA.

${ }^{4}$ BioNEM-Center of Bio-/Nanotechnology and -/Engineering for Medicine, University of Magna Graecia, 88100, Catanzaro, Italy.

${ }^{5}$ Department of Experimental Therapeutic and Rice University, Department of Biomedical Engineering, The University of Texas M. D. Anderson Cancer Center, Houston, Texas 77030, USA.

${ }^{6}$ To whom correspondence should be addressed. (e-mail: Paolo. Decuzzi@uth.tmc.edu)
}

multi-functionality). Moreover, the particle surface can be conjugated with several antibodies and ligand molecules to enhance the specific recognition of the biological target through the formation of ligand-receptor bonds, as well as polymer chains of different length and type to effectively "camouflage" the particle from the reticular-endothelial system (RES) sequestration (Fig. 1).

So far, the main strategy followed for the delivery of particulate systems has relied on exploiting the well known enhanced retention and permeability $(E P R)$ effect, in which sufficiently small particles can passively cross the tumor endothelial barrier through fenestrations with a characteristic size of the order of few hundreds of nanometers. This strategy poses a limit to the maximum size of the particles to about 100-300 nm, because larger particles would be less likely to pass through the fenestrations passively. Moreover, such an approach relies exclusively on the presence of vascular fenestrations whose size is known to change over time, being negligibly small at an early stage of the disease; to be affected by the type of tumor and site of tumor growth (4); and to be potentially reduced by anti-angiogenic therapies.

Recent studies are confirming that biological and biophysical differences exist between normal and abnormal endothelium, for cancer as well as for other diseases. Differences have been observed in the organization of the vascular network and mean blood velocity (5); and in the expression of specific markers whose surface density can be much higher on the abnormal compared to the regular endothelial cells (6). Phage display libraries are currently being used to map 


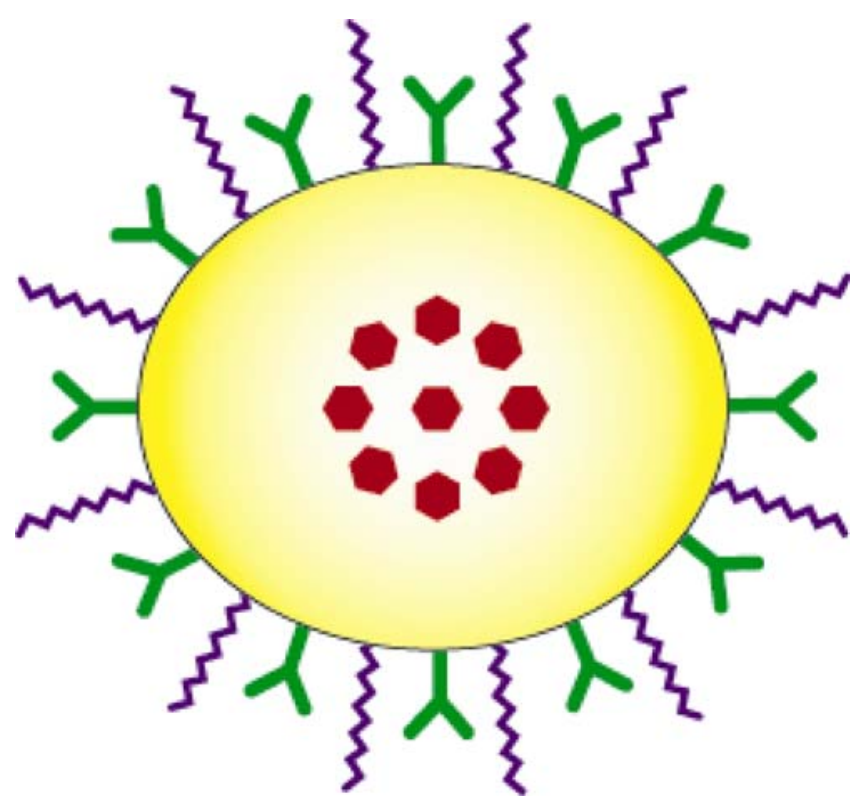

Fig. 1. A particulate system: therapeutic and contrast agents (red) are loaded within the core of the particle; the surface is decorated with ligand molecules (green) and polymer chains (violet) for RES camouflage.

the endothelial surface in-vivo targeting specific vascular markers for different diseases (7). Such experimental evidence has encouraged the definition of an alternative delivery strategy which aims at exploiting vascular biodiversity to optimally design particulate systems able to recognize the abnormal endothelium and adhere firmly to it under flow. From this privileged position, the vascular target particle (first stage) can release towards the extravascular space smaller nanoparticles (second or multiple stages) that can be loaded with therapeutic and imaging agents (8). This approach, known as vascular targeting, has two main advantages compared to the EPR based strategies: (1) can be employed for any pathology involving the endothelium, without requiring any alteration in the blood vessel permeability; (2) large particles with much higher payloads can be used.

The archetypal form of particulate systems for systemic drug delivery is the liposome, a system composed by a phospholipid and cholesterol bilayer with an aqueous inner core, which assumes a classical spherical shape according to surface energy minimization. However, striking new developments in polymer science and in micro/nano fabrication have led to the synthesis and fabrication of particulate systems with a broad spectrum of shapes. By particulate systems all the particles with a micrometer characteristic size are identified. For instance, we have demonstrated how to fabricate silicon particles with a quasi-hemispherical shape $(9,10)$; targeted networks of gold nanoparticles and bacteriophage have been developed by the group of Pasqualini and Arap (11); Illing et al. (12) have presented solid lipid nanoparticles with an anisometric shape; van Dillen et al. (13) have used ion beam radiation to transform spherical silica particles into oblate ellipsoids; Kohli and Martin (14) have presented cylindrical smart nanotubes for biotechnological applications; Subramaniam et al. (15) demonstrated that gas bubbles and liquid drops can exist in a stable non-spherical shapes; DeSimone's group (2) has developed the PRINT technology (Particle
Replication In Non-wetting Templates) for the direct fabrication of shape-specific polymer nanoparticles; and more recently Mitragotri and his colleagues (16) have demonstrated polymeric micro- and nano-particles of complex shapes. The above list just gives a glimpse of the variety of shapes and materials used to fabricate new particulate systems in which at least one geometrical feature is sub-micrometer in size.

The shift of the EPR paradigm, which has been limiting particle size in cancer therapy and imaging, in favor of the more general vascular targeting approach and the development of processes for fabricating particles with shapes other than spherical are opening the path to new, and hopefully revolutionary, design solutions where particulate systems with sizes ranging from few tens of nanometers up to few microns, and with diverse shapes, as discoidal, spheroidal, cylindrical and conical, are being employed. There is no doubt that this advance would widen the spectrum of solutions by just adding extra degrees of freedom to the problem (i.e., size and shape), but some central questions remain: Does particle geometry really matter in systemic drug delivery? Is it equally relevant at each different length scales involved in systemic drug delivery: vascular and cellular, and sub-cellular level? The motivation for this manuscript originates from these open questions.

Interestingly, within the engineering and physical community, size and shape have been known for a long time to 'matter' even at the micro- and nano-scales. For instance, Albert Einstein (17) in 1911 showed how the coefficient of molecular diffusion $D$ of a passive suspension of particles grows with the inverse of the particle radius $a$. George Jeffery (18) in 1922, studied the motion of ellipsoidal particles in viscous flows demonstrating the effect of particle shape on the rheology of the suspension. More recently, Han et al. (19) have analyzed experimentally and theoretically the Brownian motion of an ellipsoidal particle showing how $D$ as well as the particle trajectory are influenced by size and aspect ratio (shape). Moving to adhesive interactions, which dominate the micro- and nano-world, nature has donated to insects and lizard the ability of clinging vertical walls and even ceilings and being able to hold their own weight when adhering to the ceiling. A striking example is given by the geckos, whose feet are made up by a huge number of fine fibers with a terminal size of few hundreds of nanometers and with a spatula-like shape. It has been confirmed theoretically $(20,21)$ that, even at sub-micrometric scales, the shape of two interacting bodies can dramatically affect the interfacial adhesive interactions.

\section{VASCULAR DIVERSITY AND PHAGE DISPLAY LIBRARIES}

The search for molecular targets in proliferating vasculature represents a challenge. The need for such differentially-expressed endothelial cell surface markers is clear, as they are likely to play a regulatory role in angiogenesis and tumor progression. Probing molecular diversity at the cellular level is also required for the development of targeted therapies. In vivo screenings based on intravenous administration of combinatorial libraries often results in multiple targeting ligands that recognize markers expressed within selective vascular beds. By using this approach, one can dissect vascular heterogeneity within healthy or diseased tissues. 
Targeting and characterization of the receptors recognized by homing peptides has brought functional insights in vascular biology. Strategies for targeted tissue ablation have also been developed based on this approach, and utilized successfully in pre-clinical models (7).

Filamentous phage (f1, fd, and M13) are single-stranded DNA viruses that have nanometer dimensions. The major coat proteins ( $\mathrm{pIII}$ and $\mathrm{pVIII}$ ) expressed on the surface can be genetically manipulated to externally display small peptides in combinatorial libraries. The potential range of applications for this technique is quite broad, and the past decade has seen considerable progress in the construction of phage-displayed peptide libraries and in the development of screening methods in which the libraries are used to isolate peptide ligands. For example, the use of peptide libraries has made it possible to characterize interacting sites and receptorligand binding motifs within many proteins, such as antibodies involved in inflammatory reactions or integrins that mediate cellular adherence. This methodology has also been used to identify novel peptide ligands that serve as leads for the development of peptidomimetic drugs or imaging agents. In addition to peptides, larger protein domains such as singlechain antibodies can also be displayed on the phage surface. Briefly, filamentous phage are propagated in pilus-positive bacteria that are not lysed by the phage but rather secrete multiple copies of phage that display a particular insert. Phage that bind to a target molecule can be eluted and then amplified in a host bacteria. Multiple rounds of biopanning leads to a population of selective targeting peptides. The amino acid sequence of the recovered peptides is determined by sequencing the DNA corresponding to the peptide insert in the phage genome. Sequences are analyzed to monitor enriched motifs compared to the unselected library and are used to identify proteins, or ligands, with which the motifs share sequence homology, and cross-referenced with data from proteomic or microarray studies for selectivity. Once a candidate target is identified as the receptor of a homing peptide, it can be isolated, purified and cloned by using standard biochemical methods such as affinity-chromatography or genetic methods such as expression cloning.

By applying in vivo selection of peptides from phagedisplay peptide libraries, peptides that selectively target normal organs in mice and in patients have identified; so far, brain, kidney, adipose, lung, skin, pancreas, retina, intestine, uterus, prostate, and adrenal gland have been targeted. These results indicate that the vascular endothelium of normal organs is modified in ways that allow differential targeting with peptide probes. Means of identifying peptides that home to the angiogenic vasculature of tumors have been also devised. Moreover, we have assembled a panel of peptide motifs that target the blood vessels of tumor xenografts. The RGD-4C peptide has previously been identified as selectively binding $\alpha \mathrm{v}$ integrins and is now among the most studied tumor homing peptides (Table I). These tumor-targeted peptides have been shown to be more effective and less toxic than parental compounds in experimental models when coupled to cytotoxic drugs or proapoptotic peptides.

\section{SIZE AND SHAPE EFFECTS IN SYSTEMIC DELIVERY OF PARTICULATE SYSTEMS}

In the more general vascular targeting approach, particles are transported by the blood flow and interact specifically (formation of ligand-receptor bonds) and non-specifically (van der Waals, electrostatic and steric interactions) with the blood vessel walls, seeking for the diseased endothelium (vascular target). Once suitable conditions are met, in terms of expression of vascular receptors and hydrodynamic shear stresses at the wall, particles adhere firmly to the blood vessel walls and are then required to control cell uptake, avoiding or favoring it depending on their final objective. Based on this description, the intravascular "journey" of the particle can be broken down into three events, namely: the margination dynamics, the firm adhesion and the control of internalization. The role played by particle geometry (size and shape) in each of these events is discussed.

To be effective-regardless of the strategy consideredintravascularly administered particles must avoid recognition and sequestration by the reticulo-endothelial system (RES), a complex system of phagocytic cells lining the sinusoids of the liver; accumulating in the red pulp of the spleen, bone marrow and lymph nodes. Particle size has been shown to have a dramatic effect on RES sequestration: particles of $10 \mu \mathrm{m}$ and more have been shown to cause embolization in the liver and lungs (22); whereas 3-4 $\mu \mathrm{m}$ particles have been shown to accuulate permanently into the open circulation of

Table I. Cell Surface Receptors and Homing Motifs Isolated by In Vivo Phage Display

\begin{tabular}{llll}
\multicolumn{1}{c}{ Receptor } & \multicolumn{1}{c}{ Function/class } & Carrier & Localization \\
\hline$\alpha$ integrins & Cell adhesion & Yes & EC, tumor cells \\
Aminopeptidase N & Protease & Yes & EC, pericytes, tumor cells \\
Aminopeptidase A & Protease & N/D & Pericytes \\
NG2 & Proteoglycan & N/D & Pericytes, tumor cells \\
MMP-2/MMP-9 & Protease & Yes & EC, tumor cells \\
MDP & Protease & N/D & EC \\
Prohibitin & Chaperone/Cell growth/Survival & Yes & EC \\
FcRn/beta(2)m & Immunoglobulin transporter & N/D & Trophoblasts \\
IL-11R & Cytokine receptor & Yes & EC, tumor cells \\
\hline
\end{tabular}

MMP matrix metalloproteinase, $E C$ endothelial cells, $N / D$ not determined, $R$ arginine, $G$ glycine, $C$ cysteine, $D$ aspartic acid, $E$ glutamic acid, $V$ valine, $A$ alanine, $K$ lysine, $N$ asparagine, $P$ proline, $S$ serine, $L$ leucine, $T$ threonine, $H$ histidine, $W$ tryptophan, $F$ phenylalanine, $M D P$ membrane dipeptidase 
the spleen (23). For sub-micrometer particles, a classical strategy to increase the circulation half-time and escape the RES recognition consists in decorating the particles with polymer chains (PEG) (24).

\section{Margination Dynamics}

Margination is a well-known term in physiology conventionally used to describe the lateral drift of leukocytes and platelets from the core blood vessel towards the endothelial walls. Similarly a marginating particle is a particle designed to move preferentially in close proximity to the blood vessel walls, and margination dynamics is the process of lateral drifting of the particle from the blood vessel core to the walls. Accumulating the particles in close proximity to the blood vessel walls is highly desirable in vascular targeting, as well as in passive $E P R$ approach, for two main reasons: (1) particles can 'sense' the vessel walls for biological and biophysical diversities, as for instance the over expression of specific vascular markers or the presence of sufficiently large fenestrations through which extravasate; (2) particles can more easily leave the larger blood vessels in favor of the smaller, accumulating in a larger number within the microcirculation (Fig. 2). Whilst leukocyte and platelets margination is an active process requiring an interaction with red blood cells and the dilatation of inflamed vessels with blood flow reduction (25); particle margination can only be achieved by proper rational design.

Red blood cells (RBCs) have a behavior opposite to margination, with cells accumulating preferentially within the core of the vessels avoiding any interaction with the walls and staying in a larger portion in the macro-circulation (40-45\% in volume) compared to the micro-circulation $(20-25 \%$ in volume). The core accumulation of RBCs has long been described by Fahraeus and Lindqvist (26) and is referred to as the plasma skimming effect. An immediate consequence of this phenomenon is the formation of a 'cell free layer' in proximity of the wall, whose thickness varies with the size of the channel and mean blood velocity, being as large as few tens of microns in arterioles (100 and more $\mu \mathrm{m}$ in diameter) and few microns in capillaries [ten and more $\mu \mathrm{m}$ in diameter (27)]. Particles designed to marginate would then tend to accumulate in the cell free layer where an almost linear laminar flow is observed. The motion of spherical particles in a linear laminar flow has been described by Goldmann et al. (28) who showed how the exerted hydrodynamic forces grow with the particle radius and that no lateral drift would be

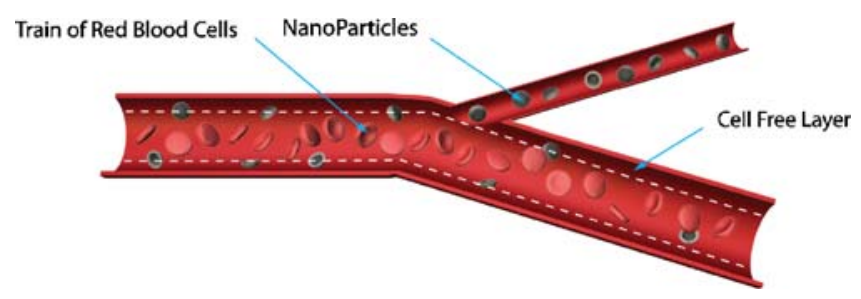

Fig. 2. Particles, rejected by the red blood cells, fast flowing in the center of the vessel, tend to accumulate in close proximity to the walls (as platelets) and more easily to leave larger vessels in favor of the smaller. This would increase particle accumulation in the micro capillary bed.

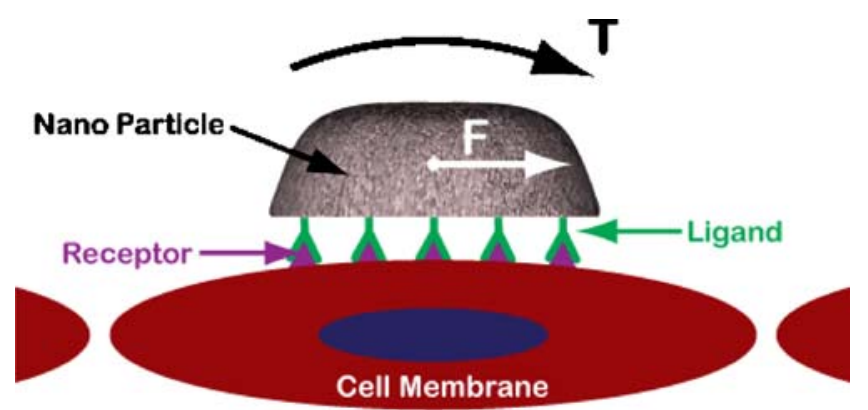

Fig. 3. The longitudinal (drag) force and the torque exerted over a particle adhering to a cell layer under flow.

observed unless an external force is applied, as gravitational or magnetic, or short ranged van der Walls and electrostatic interactions (29). In other words, a neutrally buoyant spherical particle moving in proximity to a wall can drift laterally only if an external force is applied forcing the particle to do so. It is here important to recall that gravitational force has been shown to be relevant even for sub-micrometer polystyrene beads (relative density to water of $0.05 \mathrm{~g} / \mathrm{cm}^{3}$ ), and that margination dynamics can be effectively controlled in horizontal channels by changing the size of non-buoyant nanoparticles (30).

Non-spherical particles exhibit more complex motions with tumbling and rolling which can be exploited to controll their margination dynamics without any need for lateral external forces. The longitudinal (drag) and lateral (lift) forces as well as the torque exerted by the flowing blood depend on the size, shape and orientation of the particle to the stream direction and changes over time as the particle is transported (Fig. 3). For non spherical particles, it has been shown that the lateral drifting velocity is directly related to their aspect ratio $(31,32)$, with a maximum between the two extremes: sphere, with aspect ratio unity, and disk, with aspect ratio infinity. Figure 4 shows the trajectories of a neutrally buoyant spherical and ellipsoidal particle in a straight tube mimicking a capillary: as the flow starts, the spherical particle moves to an equilibrium distance from the wall and follows the original stream line parallelly to the

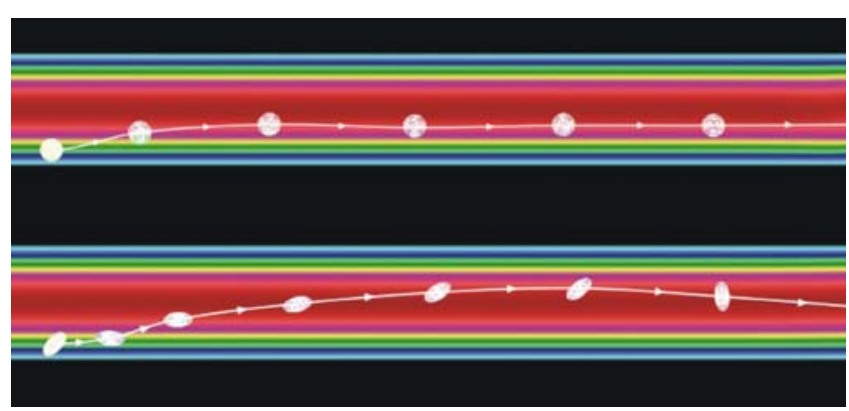

Fig. 4. The trajectory of neutrally buoyant spherical and ellipsoidal particles in an idealized capillary (straight cylindrical tube) under typical capillary hydrodynamic conditions (the ratio between the spherical particle diameter and the tube diameter is 5; the ratio between the minor axis of the ellipsoidal particle and the tube diameter is 10 and the ellipsoidal particle has an aspect ratio of 2) (32). 


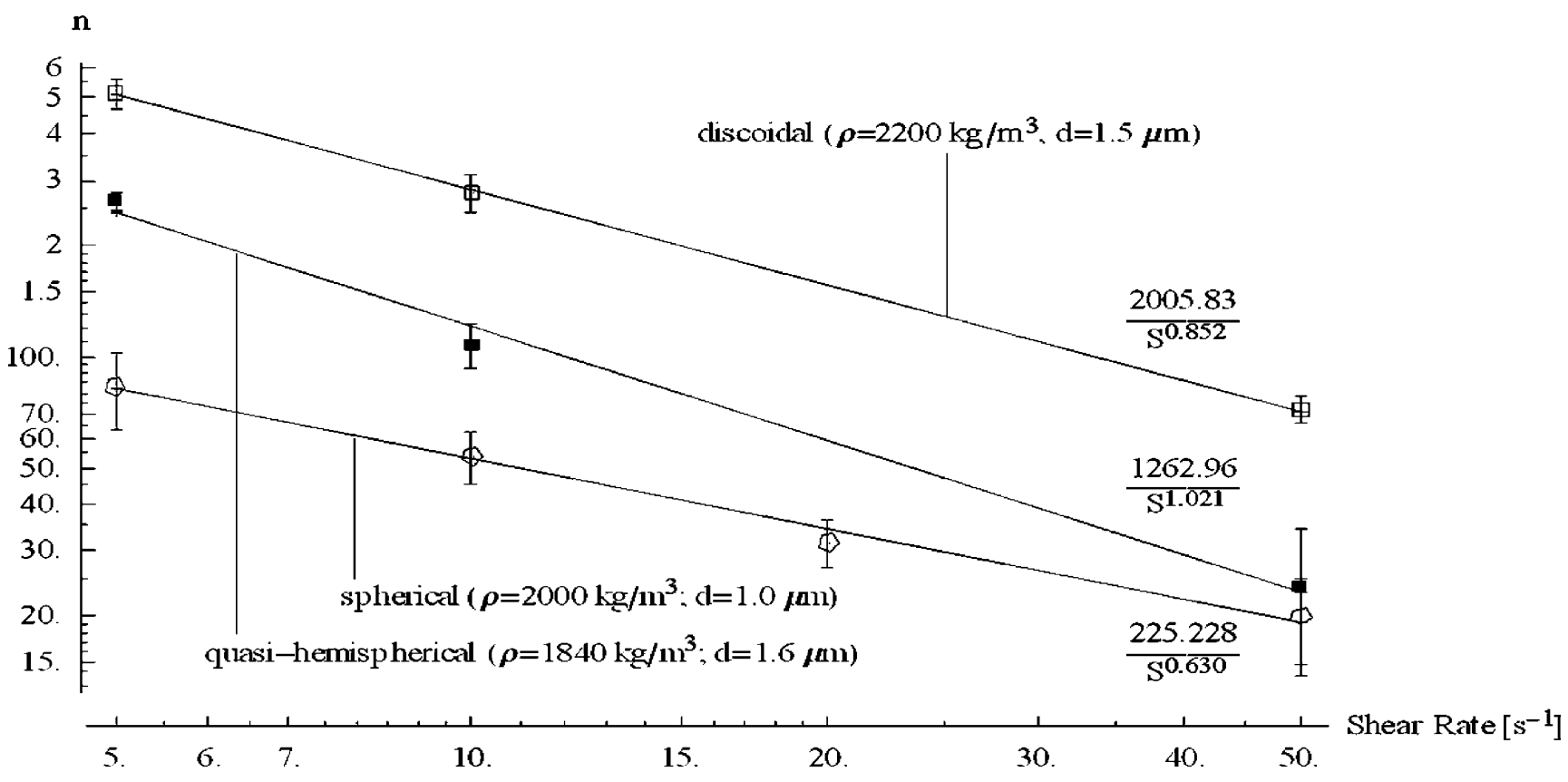

Fig. 5. The number of particles sedimenting on the bottom of a parallel plate flow chamber: the effect of different particle shapes (spherical particle: $d=1 \mu \mathrm{m}$; discoidal particle: $d=1.5 \mu \mathrm{m}$ and $h=0.3 \mu \mathrm{m}$; quasi-hemispherical particle: $d=1.6 \mu \mathrm{m}$ ).

blood vessel wall; whereas the ellipsoidal particle rotates and translates downstream drifting laterally from one side to the other of the capillary, 'exploring' the blood vessel walls. More recently, in-vitro experiments have been conducted using spherical, discoidal and quasi-hemispherical particles with the same weight injected into a parallel plate flow chamber under controlled hydrodynamic conditions (33). The experiments have shown that discoidal particles tend to marginate more than quasi-hemispherical and more than spherical particles in a gravitational field (Fig. 5). Notably, these observations neglect the interaction of the particles with blood cells, in particular RBCs. However, this is a reasonable assumption as long as the particles are sufficiently smaller than RBCs and tend to accumulate within the cell-free layer.

\section{Firm Adhesion}

The particle moving in close proximity to the blood vessels can interact both specifically and non-specifically with the endothelial cells and adhere firmly to it, once suitable conditions are met in terms of hydrodynamic shear stress at the wall and, type and level of expression of receptor molecules. From there, the particles can release their payload towards the extravascular matrix or act as vascular contrast agents in imaging applications.

Firm adhesion is ensured as long as the dislodging forces (hydrodynamic forces and any other force acting to release the particle from the target cell) are balanced by specific ligand-receptor interactions and non-specific adhesion forces arising at the cell/particle interface. The strength of adhesion can be expressed in terms of an adhesion probability $P_{\mathrm{a}}$, defined as the probability of having at least one ligandreceptor bond formed under the action of the dislodging forces. $P_{\mathrm{a}}$ is affected by the shear stress at the blood vessel wall $\mu S$, by the surface density of ligand molecules $m_{l}$ distributed over the particle surface and of receptor molecules $m_{r}$ expressed at the cell membrane, and by the size $a$ and shape (aspect ratio $\gamma$ ) of the particle (34). Interestingly, for each particle shape, a characteristic size can be identified for which the probability of adhesion has a maximum: for small particles, the hydrodynamic forces are small but the area of interaction at the particle/cell interface is also smaller leading consequently to a small number of ligand-receptor bonds involved which can not withstand even small dislodging force; for large particles, the number of ligand-receptor bonds that can be formed grows but the hydrodynamic forces grow

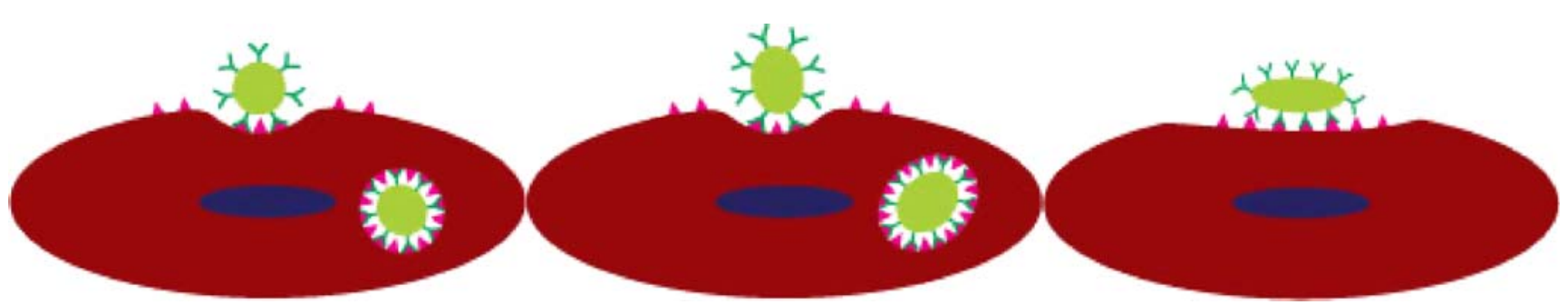

Fig. 6. Controlling cell uptake by using particles with different shapes: ovoidal and spherical particles are more easily internalized compared to elongated particles laying parallelly to the cell membrane. 

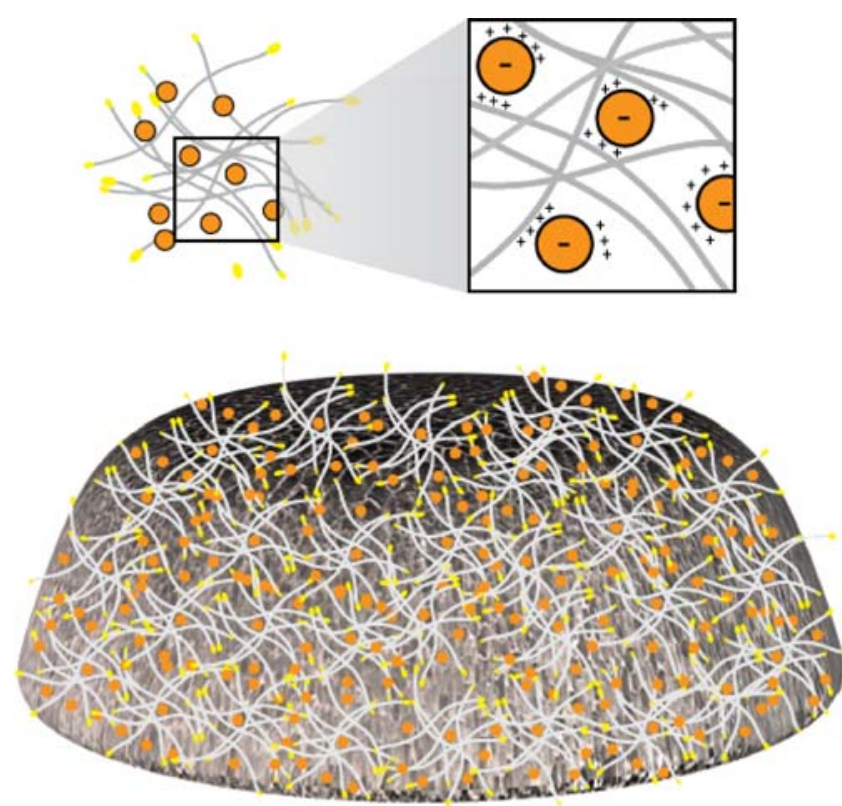

Fig. 7. A sketch of the assembly of Au-phage constructs and non spherical nano-porous silicon particles: the Au-phage construct provides the selective recognition of biological targets without altering the geometrical properties of the silicon particle.

even more. The optimal size for adhesion, that is the size for which $P_{\mathrm{a}}$ has a maximum, falls between these two limiting conditions. As an example, when considering a capillary with a shear stress at the wall of $\mu S=1 \mathrm{~Pa}$ and a surface density of receptors $m_{r}=100 \mu \mathrm{m}^{2}$, the optimal radius for a spherical particle would be of about $500 \mathrm{~nm}$ with a total volume of $0.05 \mu^{3}$, whereas the optimal volume for an oblate spheroidal particle with an aspect ratio $\gamma=2$ would be more than 50 times larger $\left(3.5 \mu^{3}\right)(34)$.

For a spherical particle, a simple relationship has been derived showing how the optimal radius $a_{\text {opt }}$ is proportional to $\mu S^{-0.72}$. Goetz and his group (35) ran in-vitro adhesion experiments employing polystyrene particles with different diameters, namely 5, 10, 15, and $20 \mu \mathrm{m}$, injected into a parallel plate flow chamber and have determined experimentally the variation of the critical shear stress $\mu S$ as a function of the particle radius $a$. A fitting of the experimental data leads to the relation which indeed is not too far from the theoretical prediction. For non-spherical particles, an explicit expression for the optimal size/volume can not be derived due to mathematical complexity, however the authors have shown that as the aspect ratio of an oblate spheroidal particle increases, the volume for which the maximum strength of adhesion is reached increases too.

\section{Control of Internalization}

Once the particle has adhered to the target cell, it should be internalized if the aim is to release drugs or therapeutic agents within the cytosol or at the nuclear level (gene delivery); or it should resist internalization if the target cell is used just as a docking site (vascular targeting) from which release second stages particles. The internalization rate is affected by the geometry of the particle.

This has been clearly shown by a number of authors for spherical particles, where the size (radius) not only affects the internalization rate, the larger is the radius and the longer is the time for cell uptake, but it also affects the internalization mechanism (36-38). In a rough estimate, small particles $(<500 \mathrm{~nm})$ are internalized by receptor mediated endocytosis, a process requiring only a local modest rearrangement of the cell cytoskeleton; large particles $(>1 \mu \mathrm{m})$ are internalized through a phagocytic process which conversely requires an extensive rearrangement of the cell cytoskeleton with the formation of protruding actin filaments; particles with an intermediate size $(>500 \mathrm{~nm}$ and $<1 \mu \mathrm{m})$ are thought to be internalized through a mixed mode. Chan's group (39) has observed, by using gold spherical particles ranging in size from 10 to $100 \mathrm{~nm}$, a maximum in the number of uptaken
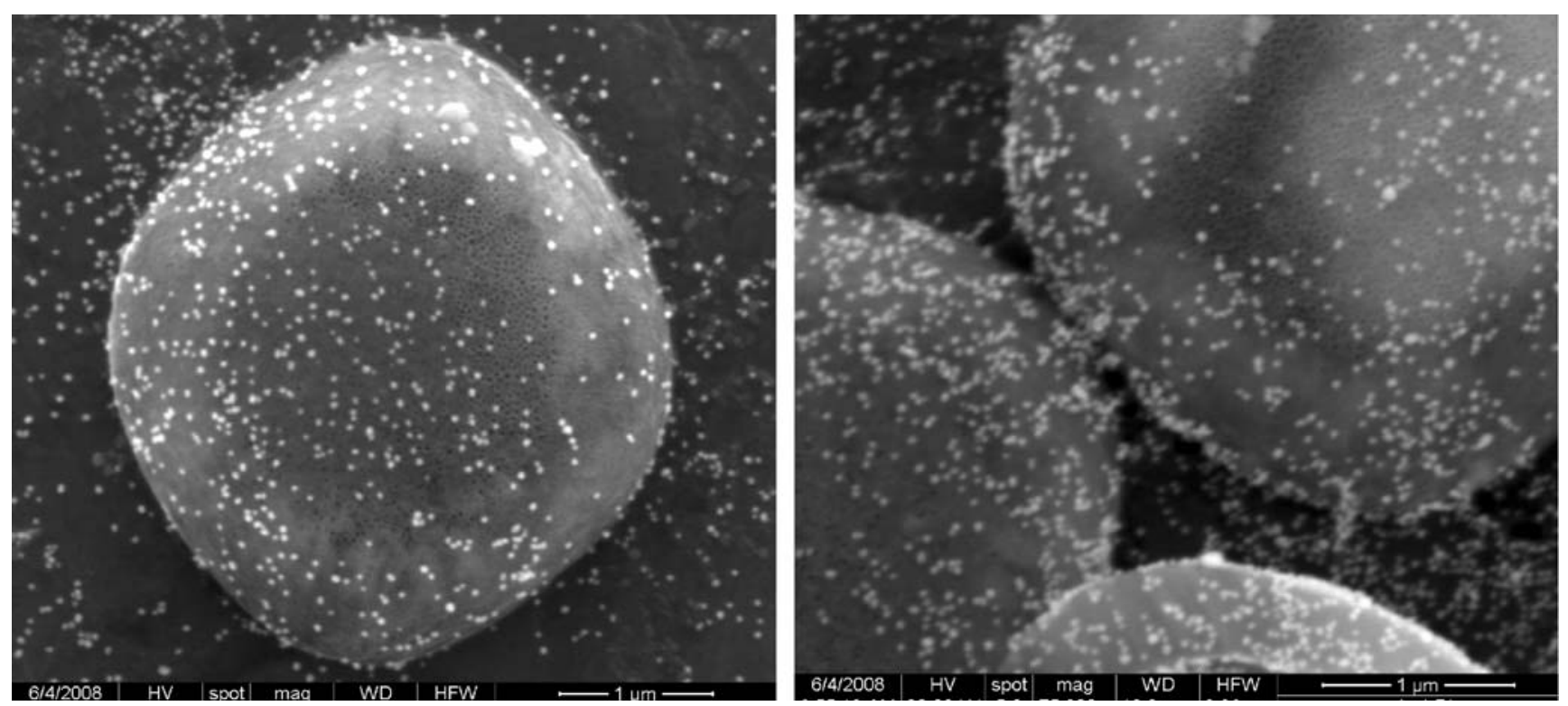

Fig. 8. A SEM image of networks of direct-assembled Au-phage scaffolds covering nano-porous microfabricated silicon particles. The shape of the silicon particle is preserved and the filamentous phage provides the exquisite targeting capability. 
particles for $50 \mathrm{~nm}$ spheres. The existance of a maximum was already observed by Osaki et al. (40). Freund and colleagues (41) developed a mathematical model for receptor-mediated endocytosis based on an energetic analysis showing that a threshold particle radius $a_{t h}$ exists below which endocytosis could never occur and, that an optimal particle radius $a_{o p t}$ exists, slightly larger than $a_{\mathrm{th}}$, for which internalization is favored with the maximum internalization rate, thus confirming theoretically the above cited experimental observations. This analysis was then generalized (30) to account for the contribution of the surface physico-chemical properties that may dramatically affect the internalization process changing significantly both $a_{\text {opt }}$ and $a_{\text {th }}$, as shown by Foged et al. (42).

More recently the effect of particle shape has been also considered by Mitragotri and his collaborators (16) for micrometer polystyrene particles phagocytosed by macrophages, and by Chan and his group (39) for gold nano-rods internalized by HeLa cells. The first group has shown in-vitro that the internalization of non-spherical particles is strongly influenced by their orientation with respect to the cell membrane. A characteristic angle $\Omega$ has been introduced varying between 0 and $90^{\circ}$, with $\Omega$ tending to zero for slender particles having their major axis aligned orthogonally to the cell membrane and tending to $90^{\circ}$ for slender particles with their major axis parallel to the cell membrane. The angle $\Omega$ is $45^{\circ}$ for a spherical particle. The rate of internalization was shown to increase with an increase in $\Omega$ tending to zero (no internalization) for angles larger than about $50^{\circ}$. At the nanoscale, Chan and collaborators have observed that gold nano-rods with large length-to-radius ratio (elongated) are internalized in a smaller portion compared to spherical particles with an equivalent characteristic size. The authors noted that this could be attributed either to the different curvature of the particles (spherical versus cylindrical) or to the different amount of molecules adsorbed onto the particle surface mediating the particle-cell interaction. More interestingly, Chan and colleagues have also shown how the particle geometry at the nanoscale can have an effect on the biological activity of the cell (43).

A more recent theoretical model has been developed by the authors for the receptor mediated endocytosis of nonspherical particles (44) showing how elongated particles laying parallelly to the cell membrane are less prone to internalization compared to spherical particles or particles laying normally to the cell membrane (Fig. 6). These results clearly show how particle size and shape can be used to control the internalization process effectively, and that particles slightly deviating from the spherical shape are more easily internalized compared to elongated particles deviating severely from the classical spherical shape.

\section{RATIONAL DESIGN OF PARTICULATE SYSTEMS}

The performance of a particulate systems administered at the systemic level is influenced by physiological and biophysical properties, as the hydrodynamic conditions at the site of adhesion and the surface density of receptor molecules, and by particle properties that can be controlled during the manufacturing process as the geometry (size and shape) and the surface features (density of ligand molecules, density and length of polymer chains, electrostatic charge).
Clearly, the number of possible combinations is extraordinarily large and thus requires a rational approach to the selection of the best particle just as in engineering optimization problems where the best solution has to be found within all feasible solutions given specific constrains and objective functions.

Recently, a mathematical model has been developed (45) that allows to predict the adhesive and endocytotic performances of particulate systems based on three different categories of governing parameters: geometrical (radius of the particle); biophysical (ligand-to-receptor surface density ratio; non-specific interaction parameter; hydrodynamic force parameter) and biological (ligand-receptor binding affinity). This finding has lead to the definition of design maps through which the three different states of the particulate system can be predicted: no adhesion at the blood vessel walls; firm adhesion with no internalization by the endothelial cells; firm adhesion and internalization.

These all emphasize the importance that both geometry and surface physico-chemical properties have in the design of particulate systems. In this respect, the integration of phage display-based combinatorial tissue targeting and microfabricated particulate systems is emerging as a powerful tool. It has been now clearly established that the assembly of phage and nanoparticles occurs spontaneously, without the need for cross-liking, conjugation or phage genetic alterations, possibly yielding a broad range of applications. For instance, biologically active networks of direct-assembled Au-phage scaffolds have already shown highly favorable properties in vivo $(11,46,47)$. These are the result of the spontaneous selfassembly of filamentous phages and $40 \mathrm{~nm}$ gold nanoparticles, and the adhesive and internalization properties of this construct have been shown to be easily tunable by changing the phage/particle ratio and the level of aggregation.

More recently, the Au-phage scaffolds have been combined with non-spherical silicon particles (Figs. 7 and 8) leading to a particulate system whit superior targeting properties (filamentous phage) and with an exquisite control on size and shape (microfabricated silicon particle).

\section{CONCLUSIONS}

The characterization of the endothelial biodiversity and the precise control of size and shape in micro/nano-fabrication have promoted a paradigm shift in the systemic administration of particulate systems for biomedical applications: both geometry and surface physico-chemical properties contribute substantially to the optimal design of the particulate system.

Geometry affects the transport and biodistribution of the particles, at the vascular level; the strength of adhesion and the internalization rate at the cellular level. Geometry favors particle accumulation in proximity of the blood vessels (margination dynamics) to better sense endothelial wall biodiversity. Geometry affects the strength of adhesion to cells improving target specificity. Geometry influences the rate of internalization discriminating between particles for nuclear delivery (rapid internalization) and particles for vascular targeting (no internalization).

In this respect, the integration of phage display-based combinatorial tissue targeting and microfabricated particulate 
systems is emerging as a powerful tool: the filamentous phage provide exquisite targeting while preserving the peculiar geometry of the microfabricated particulate.

As with the 'rational drug design' introduced within the pharmaceutical community decades ago (48), predictive mathematical models, in-vitro assays and in-vivo testing need to be integrated in one sole framework for the selection of optimal particulate systems leading to what can be baptized as the rational design of particulate systems.

\section{ACKNOWLEDGMENTS}

The authors PD and MF were supported by the U.S. Department of Defense through the grant W81XWH-04-2-0035 and by the Emerging Technology Fund by the State of Texas through the grant "Acquisition of Scientific Superiority in Biomedical Nanotechnology", and to the Department of Defense project-BioGEO: Understanding the Effect of Size and Shape in Biological Systems to Learn Fabricating Bio-Mimetic Artificial Systems with Superior Properties. MF was also supported by NASA through the grant SA23-06-017 "Nanotechnology for Space Medicine". The authors RP and WA acknowledge the support of the Marcus Foundation.

\section{REFERENCES}

1. O. C. Farokhzad, J. Cheng, B. A. Teply, I. Sherifi, S. Jon, P. W. Kantoff, J. P. Richie, and R. Langer. Targeted nanoparticleaptamer bioconjugates for cancer chemotherapy in vivo. Proc. Natl. Acad. Sci. USA. 103:6315-6320 (2006). doi:10.1073/ pnas.0601755103.

2. J. P. Rolland, B. W. Maynor, L. E. Euliss, A. E. Exner, G. M. Denison, and J. M. DeSimone. Direct fabrication and harvesting of monodisperse, shape-specific nanobiomaterials. J. Am. Chem. Soc. 127(28):10096-10100 (2005). doi:10.1021/ja051977c.

3. M. Ferrari. Cancer nanotechnology: opportunities and challenges. Nat. Rev. Cancer. 5:161-171 (2005). doi:10.1038/nrc1566.

4. S. K. Hobbs, W. L. Monsky, F. Yuan, W. G. Roberts, L. Griffith, V. P. Torchilin, and R. K. Jain. Regulation of transport pathways in tumor vessels: role of tumor type and microenvironment. Proc. Natl. Acad. Sci. USA. 95:4607-4612 (1998). doi:10.1073/ pnas.95.8.4607.

5. R. K. Jain. Transport of molecules, particles, and cells in solid tumors. Annu. Rev. Biomed. Eng. 1:241-263 (1999). doi:10.1146/ annurev.bioeng.1.1.241.

6. D. Neri, and R. Bicknell. Tumor vascular targeting. Nat. Cancer. Rev. 5:436-446 (2005). doi:10.1038/nrc1627.

7. A. Sergeeva, M. G. Kolonin, J. J. Molldrem, R. Pasqualini, and W. Arap. Display technologies: application for the discovery of drug and gene delivery agents. Adv. Drug Deliv. Rev. 58:16221654 (2006). doi:10.1016/j.addr.2006.09.018.

8. J. Sakamoto, A. Annapragada, P. Decuzzi, and M. Ferrari. Antibiological barrier nanovector technology for cancer applications. Expert Opin. Drug Deliv. 4:359-369 (2007). doi:10.1517/ 17425247.4.4.359.

9. M. H. Cohen, K. Melnik, A. A. Boiarski, M. Ferrari, and F. J. Martin. Microfabrication of silicon-based nanoporous particulates for medical applications. Biomed. Microdev. 5:253-259 (2003). doi:10.1023/A:1025768411300.

10. E. Tasciotti, K. Plant, R. Bhavane, X. Liu, A. D. Leonard, B. K Price, M. Cheng, P. Decuzzi, J. M. Tour, F. Robertson, and M. Ferrari. Nanoporous silicon particles as a multistage delivery system for imaging and therapeutic applications. Nat. Nanotechnol. 3:151-157 (2008). doi:10.1038/nnano.2008.34.

11. G. R. Souza, D. R. Christianson, F. I. Staquicini, M. G. Ozawa, E. Y. Snyder, R. L. Sidman, J. H. Miller, W. Arap, and R.
Pasqualini. Networks of gold nanoparticles and bacteriophage as biological sensors and cell-targeting agents. Proc. Natl. Acad. Sci. USA. 103:1215-1220 (2006). doi:10.1073/pnas.0509739103.

12. A. Illing, T. Unruh, and M. H. Koch. Investigation on particle selfassembly in solid lipid-based colloidal drug carrier systems. Pharm. Res. 21:592-597 (2004). doi:10.1023/B:PHAM.0000022405.49805.a7.

13. T. van Dillen, A. van Blaaderen, and A. Polman. Ion beam shaping of colloidal assemblies. Materials Today. 7:40-46 (2004). doi:10.1016/S1369-7021(04)00345-1.

14. P. Kohli, and C. R. Martin. Smart nanotubes for biotechnology. Curr Pharm Biotechnol. 6:35-47 (2005).

15. A. B. Subramaniam, M. Abkarian, L. Mahadevan, and H. A. Stone. Non-spherical bubbles. Nature. 438:930 (2005). doi:10.1038/438930a.

16. J. A. Champion, Y. K. Katare, and S. Mitragotri. Making polymeric micro- and nanoparticles of complex shapes. Proc. Natl. Acad. Sci. USA. 104:11901-11904 (2007). doi:10.1073/ pnas.0705326104.

17. A. Einstein. Elementary observations on thermal molecular motion in solids (Elementare Betrachtungen über die thermische Molekularbewegung in festen Körpern). Annalen der Physik. 35:679-694 (1911)

18. G. B. Jeffery. The motion of ellipsoidal particles immersed in a viscous fluid. Proc. Royal Soc. London. Series A. 102:161-179 (1922). doi:10.1098/rspa.1922.0078.

19. Y. Han, A. M. Alsayed, M. Nobili, J. Zhang, T. C. Lubensky, and A. G. Yodh. Brownian motion of an ellipsoid. Science. 314:626630 (2006). doi:10.1126/science.1130146.

20. R. Gissi, and P. Decuzzi. The effect of shape and size in micronanodimples adhesion. J. Appl. Phys. 98:14310 (2005). doi:10.1063/1.1944907.

21. R. Spolenak, S. Gorb, H. J. Gao, and E. Arzt. Effects of contact shape on the scaling of biological attachments. Proc. R. Soc. A. 461:305-319 (2005). doi:10.1098/rspa.2004.1326.

22. L. Illum, and S. S. Davis. The targeting of drugs parenterally by use of microspheres. J. Parenter. Sci. Tech. 36:242-248 (1982).

23. L.-T. Chen. Microcirculation of the spleen: An open or closed circulation. Science. 201:157-159 (1978). doi:10.1126/science. 663644.

24. S. M. Moghimi, A. C. Hunter, and J. C. Murray. Nanomedicine: current status and future prospects. FASEB J. 19:311-330 (2005). doi:10.1096/fj.04-2747rev.

25. H. L. Goldsmith, and S. Spain. Margination of leukocytes in blood flow through small tubes. Microvasc. Res. 27:204-222 (1984). doi:10.1016/0026-2862(84)90054-2.

26. R. Fahraeus, and T. Lindqvist. The viscosity of the blood in narrow capillary tubes. Am. J. Physiol. 96:562-568 (1931).

27. M. Sharan, and A. S. Popel. A two-phase model for flow of blood in narrow tubes with increased effective viscosity near the wall. Biorheology. 38:415-428 (2001).

28. A. J. Goldman, R. G. Cox, and H. Brenner. Slow viscous motion of a sphere parallel to a plane wall. II. Couette flow. Chem. Eng. Sci. 22:653 (1967). doi:10.1016/0009-2509(67)80048-4.

29. P. Decuzzi, S. Lee, B. Bhushan, and M. Ferrari. A theoretical model for the margination of particles within blood vessels. Ann. Biomed. Eng. 33:179-190 (2005). doi:10.1007/s10439-005-8976-5.

30. P. Decuzzi, and M. Ferrari. The role of specific and non-specific interactions in receptor-mediated endocytosis of nanoparticles. Biomaterials. 28:2915-2922 (2007). doi:10.1016/j.biomaterials. 2007.02.013.

31. E. Gavze, and M. Shapiro. Motion of inertial spheroidal particles in a shear flow near a solid wall with special application to aerosol transport in microgravity. J. Fluid Mech. 371:59-79 (1998). doi:10.1017/S0022112098002109.

32. N. Filipovic, B. Stojanovic, N. Kojic, and M. Kojic. Computer modeling in bioengineering-theoretical background. Examples and Software. J. Wiley and Sons, Wiley-Blackwell, 2008.

33. F. Gentile, C. Chiappini, D. Fine, R. C. Bhavane, M. S. Peluccio, M. Ming-Cheng Cheng, X. Liu, M. Ferrari, and P. Decuzzi. Scaling laws in the margination dynamics of non-spherical inertial particles in a microchannel. J. Biomech. [Jun 18. Epub ahead of print] (2008).

34. P. Decuzzi, and M. Ferrari. The adhesive strength of nonspherical particles mediated by specific interactions. Biomaterials. 27:5307-5314 (2006). doi:10.1016/j.biomaterials.2006.05.024. 
35. V. R. Shinde Patil, C. J. Campbell, Y. H. Yun, S. M. Slack, and D. J. Goetz. Particle diameter influences adhesion under flow. Biophys J. 80:1733-1743 (2001).

36. J. Rejman, V. Oberle, I. S. Zuhorn, and D. Hoekstra. Sizedependent internalization of particles via the pathways of clathrinand caveolae-mediated endocytosis. Biochem. J. 377:159-169 (2004). doi:10.1042/BJ20031253.

37. M. Koval, K. Preiter, C. Adles, P.D. Stahl, and T. H. Steinberg. Size of IgG-Opsonized Particles Determines Macrophage Response during Internalization. Experimental Cell Res. 242:265273 (1998). doi:10.1006/excr.1998.4110.

38. M. Herant, V. Heinrich, and M. Dembo. Mechanics of neutrophil phagocytosis: experiments and quantitative models. J. Cell. Sci. 119:1903-1913 (2006). doi:10.1242/jcs.02876.

39. B. D. Chithrani, A. A. Ghazani, and W. C. Chan. Determining the size and shape dependence of gold nanoparticle uptake into mammalian cells. Nano Lett. 6:662-668 (2006). doi:10.1021/ n1052396o.

40. F. Osaki, T. Kanamori, S. Sando, T. Sera, and Y. Aoyana. J. Am. Chem. Soc. 126:6520-6521 (2004). doi:10.1021/ja048792a.

41. H. Gao, W. Shi, and L. B. Freund. Mechanics of receptormediated endocytosis. Proc. Natl. Acad. Sci. USA. 102:9469-9474 (2005). doi:10.1073/pnas.0503879102.
42. C. Foged, B. Brodin, S. Frokjaer, and A. Sundblad. Particle size and surface charge affect particle uptake by human dendritic cells in an in vitro model. Int. J. Pharm. 25:315-322 (2005). doi:10.1016/j.ijpharm.2005.03.035.

43. M. Ferrari. Nanogeometry: Beyond drug delivery. Nat. Nanotechnol. 3:131-132 (2008). doi:10.1038/nnano.2008.46.

44. P. Decuzzi, and M. Ferrari. The receptor-mediated endocytosis of nonspherical particles. Biophys. J. 94:3790-3797 (2008). doi:10.1529/biophysj.107.120238.

45. P. Decuzzi, and M. Ferrari. Design maps for nanoparticles targeting the diseased microvasculature. Biomater. 29:377-384 (2008).

46. G. R. Souza, C. S. Levin, A. Hajitou, E. Pasqualini, W. Arap, and J. H. Miller. In vivo detection of gold-imidazole self-assembly complexes: NIR-SERS signal reporters. Anal. Chem. 78:62326237 (2006b). doi:10.1021/ac060483a.

47. G. R. Souza, E. Yonel-Gumruk, D. Fan, J. Easley, R. Rangel, L. Guzman-Rojas, J. H. Miller, W. Arap, and E. Pasqualini. Bottom-up assembly of hydrogels from bacteriophage and au nanoparticles: the effect of cis- and trans-acting factors. PLoS ONE. 3:e2242 (2008). doi:10.1371/journal.pone.0002242.

48. C. Heidelberger. Cancer chemotherapy with purine and pyrimidine analogues. Ann. Rev. Pharmacol. 7:101-124 (1967). doi:10.1146/annurev.pa.07.040167.000533. 